\title{
Melting of Monolayer Xenon on Silver: The Hexatic Phase in the Weak-Substrate Limit
}

\author{
N. Greiser, G. A. Held, R. Frahm, ${ }^{(a)}$ R. L. Greene, and P. M. Horn \\ IBM Thomas J. Watson Research Center, Yorktown Heights, New York 10598 \\ and \\ R. M. Suter \\ Department of Physics, Carnegie-Mellon University, Pittsburgh, Pennsylvania 15213 \\ (Received 10 June 1987)
}

\begin{abstract}
We report the results of a high-resolution synchrotron $\mathrm{x}$-ray-scattering study of the melting of Xe monolayers adsorbed on $\mathrm{Ag}(111)$. We find that at high temperatures $(T>100 \mathrm{~K})$ all features of the melting transition are, within errors, the same as those found for xenon on graphite. Specifically, in both cases the xenon solid melts into a "hexatic phase" with essentially identical hexatic stiffness. This strongly suggests that the existence of the hexatic phase is a feature of the two-dimensional melting process rather than the adsorbate-substrate interaction.

PACS numbers: $64.70 . \mathrm{Dv}, 05.70 . \mathrm{Fh}, 64.60 .-\mathrm{i}$
\end{abstract}

The nature of the two-dimensional (2D) melting transition remains both theoretically and experimentally controversial. On the one hand, an elegant theory based on the unbinding of topological defects suggests that melting could occur via two continuous transitions: First, the 2D solid evolves into an orientationally ordered, positionally disordered fluid (or hexatic) phase, and subsequently, this hexatic phase melts into an isotropic liquid. ${ }^{1}$ Various experiments have confirmed hexatic order in liquids, ${ }^{2-4}$ and high-resolution x-ray-scattering studies ${ }^{5}$ of xenon on graphite have displayed continuous melting transitions with fluid correlation lengths exceeding 2000 $\AA$. High-precision thermodynamic measurements of the xenon/graphite system indicate a crossover from a very weakly first-order transition to a continuous one at high temperatures. ${ }^{6}$ Alternately, some theories predict that 2D melting is inherently a first-order transition. ${ }^{7}$ Furthermore, computer simulations of two-dimensional melting are often interpreted as proving that the substratefree transition is first order. ${ }^{8}$

One significant difference between most computer simulations and the experiments on adsorbed layers is the corrugation of the substrate adsorption potential. For incommensurate overlayers, the corrugation energy tends to orientationally align the axes of the adsorbate lattice with respect to specific substrate directions. ${ }^{9}$ For sixfold-symmetric surfaces, this interaction generates the field conjugate to the hexatic order parameter. ${ }^{1,5}$ Therefore, the hexatic order observed for rare gases on graphite might be partially or totally due to the adsorbategraphite interaction. Furthermore, the substrate field may suppress orientational fluctuations driving an otherwise first-order transition to be continuous. ${ }^{5}$

To probe the role of the substrate in the melting process, we have carried out a detailed high-resolution $\mathrm{x}$ ray-scattering study of xenon on silver (111). Ag(111) was chosen for a number of reasons: Firstly, $\operatorname{Ag}(111)$ is a stable, easy to clean surface which can be made with relatively large step-free regions. ${ }^{10}$ Second, xenon on silver has been studied extensively both experimentally ${ }^{10}$ and theoretically. ${ }^{11}$ Low-temperature LEED studies show that the xenon monolayers form an incommensurate lattice which is orientationally aligned with respect to the silver substrate. ${ }^{10}$ The alignment is such that the xenon reciprocal lattice is collinear with the silver lattice (i.e., rotated $30^{\circ}$ from the xenon on graphite case). Finally, $\mathrm{Ag}(111)$ is a close-packed triangular face with a high density of conduction electrons which smooth the repulsive part of the corrugation potential. Atomscattering studies show that the corrugation potential of $\mathrm{Ag}(111)$ is 0.1 times that of graphite; this suggests that the orientational field for $\mathrm{Xe}$ on $\mathrm{Ag}(111)$ is an order of magnitude smaller than that of xenon on graphite. ${ }^{10-12}$ Indeed, it is generally believed that this potential is so small that the xenon orientation is determined by surface steps rather than the substrate corrugation. ${ }^{12}$ Therefore, the melting of the xenon on silver represents an archetypal example of two-dimensional melting in the weaksubstrate limit.

In order to carry out this experiment, we were compelled to construct a unique UHV apparatus which allowed for low-temperature $(T \gtrsim 50 \mathrm{~K}) \mathrm{x}$-ray scattering with the sample in equilibrium with high three-dimensional xenon gas pressures $(P \lesssim 100$ Torr $)$. Briefly, a UHV chamber with a cylindrical 1.5-in.-diam Be window was mounted on the cold finger of a Displex cryostat. The design allowed the entire central section of the chamber, including the sample and all exterior walls to be cooled. An outer evacuated region, contained within a 5-in.-diam Be window shroud, served to isolate the cold central section from room temperature. A sputter gun could be inserted into the cold region of the chamber through two Be-Cu leaf spring windows. The chamber itself was evacuated with a small ion pump while the 
external lines and gas-handling system utilized a turbo pump. The entire Displex-chamber assembly was mounted onto a Huber six-circle goniometer.

The Ag sample was aligned, spark cut, mechanically polished, and etched. The in-plane substrate mosaic spread was about $0.16^{\circ}$ and the surface was aligned to the [1 111 ] direction to better than $0.1^{\circ}$. Before installation into the cryostat, the sample was mounted into a UHV chamber within which it was sputtered and annealed until the concentrations of sulfur, carbon, and oxygen diffusing to the surface remained below Auger detection limits. The sample was then mounted into the scattering chamber where it was again repeatedly sputtered and annealed.

We now discuss the scattering experiment. The experiment was carried out at IBM-MIT beam line X-20A at the National Synchrotron Light Source. The synchrotron beam was focused on the sample with a platinumcoated silicon mirror and monochromated to $9.3 \mathrm{keV}$ with silicon (111) crystals. The transverse resolution within the scattering plane was about $7 \times 10^{-5} \AA^{-1}$ HWHM. The direction of the scattered $x$ rays was determined by slits set such that the longitudinal instrumental resolution was $0.008 \AA^{-1} \mathrm{HWHM}$, approximately equal to the longitudinal width of the xenon $(1,0)$ surface peak.

After the sample was flashed clean, a fixed pressure of xenon was admitted into the cell and pairs of radial $(\theta$ $2 \theta)$ and angular $x$-ray scans were taken through a xenon $(1,0)$ surface peak. These peaks were incommensurate with, and aligned colinear to, the Ag substrate. Typical results are shown in Fig. 1. The angular scan data have been corrected for incident-beam absorption for $\theta \gtrsim 0$. At each fixed pressure, the xenon scattering typically evolves as illustrated by the $P=1.5$ Torr data shown in Fig. 2 , where we plot the radial $(\kappa)$ and the angular $\left(\kappa_{\theta}\right)$

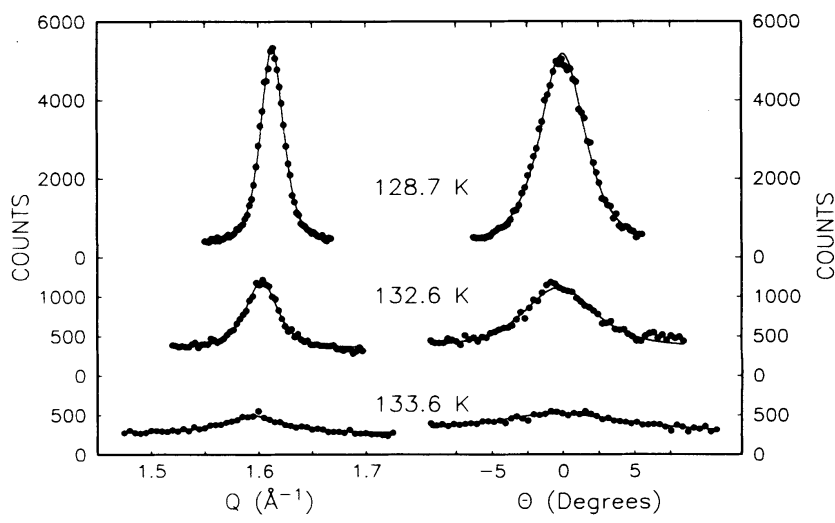

FIG. 1. Radial (left) and angular (right) scans through the xenon $(1,0)$ peak position at a pressure of 1.5 Torr and at various temperatures. The solid lines through the data are best fits to a Lorentzian-squared functional form. half-widths (corrected for instrumental resolution and substrate mosaic) versus temperature. At low temperatures, the width of the xenon peak remains fixed in both the angular and radial directions. (Typical widths are $\simeq 0.01 \AA^{-1}$ HWHM radial and $\simeq 2^{\circ} \doteq 0.05 \AA^{-1}$ HWHM angular, although the exact numbers varied with the details of the surface preparation.) At a critical temperature, $T_{M}(P)$ the xenon layer melts and both widths evolve rapidly as shown in Fig. 2 . Both $T_{M}(P)$ and the lattice constant at melting $Q_{M}(P)$ are, within experimental error, identical to that found for the melting of xenon on graphite. For example, at $P=1.5$ Torr we find $T_{M}=131.8 \mathrm{~K}$ and $Q_{M}=1.602 \AA^{-1}$ compared to $132.5 \mathrm{~K}$ and $1.592 \AA^{-1}$ as estimated from the data of Dimon et al. ${ }^{13}$ and $131.3 \mathrm{~K}$ as estimated from Colella and Suter. ${ }^{6}$

Empirically we find that a Lorentzian-squared line shape accurately fits all our scans as illustrated in Fig. 1. Best fits to the data are shown as the solid lines. The fact that the solid Bragg peaks evolve slowly into broadened liquid spots (see Fig. 2) with the sixfold pattern of the solid implies that the xenon liquid has hexatic long-range order; a normal liquid would display a ring of

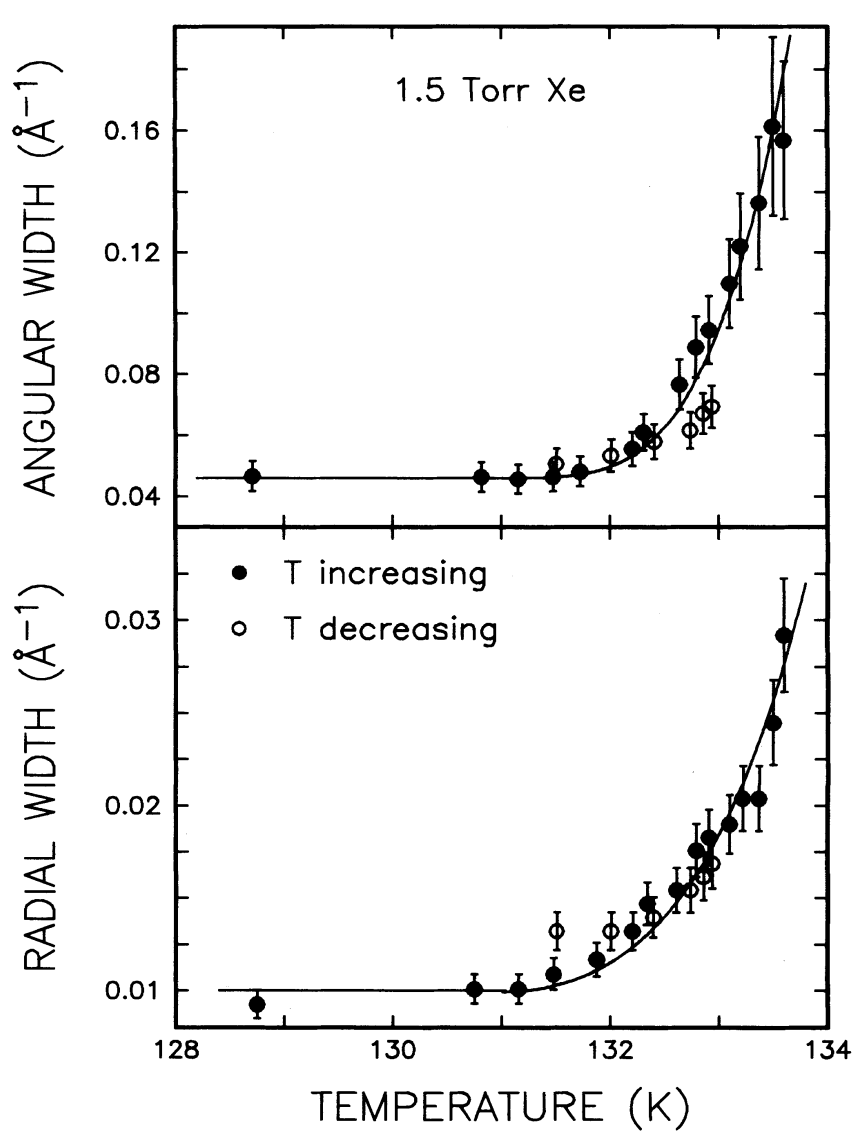

FIG. 2. Half-widths of the radial $(\kappa)$ and angular $\left(\kappa_{\theta}\right)$ scans as a function of temperature. 
scattering. ${ }^{4}$ The observation of sharp spots in the liquid phase near the transition implies the existence of a large positional correlation length $\xi=1 / \kappa$. We see no evidence for the coexistence line shapes characteristic of a firstorder transition. The aspect ratio of the spot is a measure of the hexatic stiffness to bond-angle fluctuations in the liquid phase. ${ }^{4,5}$ We quantify this in Fig. 3, where we plot $\left(\kappa_{\theta}-\kappa\right) / \kappa$, the excess angular width divided by the radial spot width. $\left(\kappa_{\theta}-\kappa\right) / \kappa=0$ for perfect bondorientational order and $\left(\kappa_{\theta}-\kappa\right) / \kappa \rightarrow \infty$ for a liquid.

Our experimental results are best understood within the context of the Hamiltonian for the adsorbed xenon fluid,

$$
\mathcal{H}=\frac{1}{2} K_{A} \int(\nabla \theta)^{2} d^{2} r+\int h_{6}[1-\cos 6(\theta-\tilde{\theta})] d^{2} r+\int E_{P}[1-\cos (6 \theta)] \delta\left(\mathbf{r}-\mathbf{r}_{s}\right) d^{2} r .
$$

The first two terms are the usual hexatic Hamiltonian discussed previously, ${ }^{1,4,5}$ where $K_{A}$ is the hexatic stiffness to bond-angle $(\theta)$ fluctuations, and $h_{6}$ is the substrate orientational field which tends to orient the adsorbed layer to an angle $\tilde{\theta}$. The third term represents orientational pinning at $\mathbf{r}=\mathbf{r}_{s}$ along the edges or steps on the substrate surface and favors $\theta=0^{\circ}$. The pinning energy per edge length is $E_{P}$ which, in the strong pinning limit, can be no greater than the energy cost per unit length to create a grain boundary of angle $\theta$. Experiments for xenon on $\mathrm{Pt}^{12}$ and theoretical estimates for orientational epitaxy energies ${ }^{14}$ suggest that $\tilde{\theta}=30^{\circ}$. Our observation of an aligned xenon solid $\left(\theta=0^{\circ}\right)$ implies, therefore, that in the solid phase, the third term in Eq. (1) dominates the second, i.e., $E_{P}>h_{6} L$, where $L$ is the linear size of our surface domains (or the distance between steps $\simeq 350$ $\AA) .{ }^{15}$ A crude $T=0 \mathrm{~K}$ estimate of the maximum allowable $E_{p}$ for xenon is about $18 \mathrm{~K}$ per atom ${ }^{16}$ suggesting that the substrate $h_{6}$ is indeed quite small. The second term in Eq. (1) gives no bond-angle stiffness near $\theta=0^{\circ}$ and for simplicity is ignored. Near $T_{M}$, small bondangle fluctuations about $\theta=0^{\circ}$ can be crudely approximated by

$$
\left\langle\delta^{2}\right\rangle=\frac{k_{\mathrm{B}} T}{2 \pi} \int \frac{q d q}{K_{A} q^{2}+36 E_{P}\left(\xi_{6} / a L\right)},
$$

where $\xi_{6}$ is the bond-angle-bond-angle correlation length and $a$ is the xenon lattice constant. Equation (2) approximates the pinning energy density $E_{P} / a$, which acts within a region of width $\xi_{6}$, by a uniform field of magnitude $E_{P} \xi_{6} / a L$, which acts throughout the surface region.

We display in Fig. 3 three models for the origin of our observed hexatic phase. Firstly, we assume that the xenon overlayer is a normal fluid with $K_{A}=0$ and $\xi_{6} \simeq \xi$. In this picture the orientational order is due entirely to the step pinning. This model leads to an orientational width $\kappa_{\theta} \sim \xi^{-3 / 2}$ and has one adjustable parameter, the renormalized pinning strength, $E_{P}\left(\xi_{6} / \xi\right)^{3}$, which was set to $90 \mathrm{~K}$ per atom to fit the data. The second model assumes that the orientation stiffness for both xenon on graphite and xenon on silver is due entirely to the substrate term $h_{6}$. This naive picture has $\kappa_{\theta} \sim \xi^{-1}$ and, if we assume $h_{6}$ (silver) $=0.1 h_{6}$ (graphite), ${ }^{10}$ has no adjustable parameters. Finally we assume that the orientational order is due to the hexatic stiffness $K_{A}$ and that the magnitude of $K_{A}$ is exactly the same as for xenon on graphite. ${ }^{5}$ This model has $\kappa_{\theta} \sim \xi^{-1}$ and fits the data with essentially no adjustable parameters.

While we cannot rule out step pinning as a partial contributor to the observed hexatic order, the quantitative agreement of the data for xenon on silver with that for xenon on graphite is remarkable. It would be an amazing coincidence if the step-pinning energy for xenon on silver had the exact magnitude necessary to reproduce the xenon-graphite stiffness. Furthermore, strong pinning without some intrinsic hexatic order would lead to a coexistence line shape arising from liquidlike scattering from the center of the platelet and hexaticlike scattering from the edges. Experimentally, we see no evidence for this.

Another interesting feature of our data is the orientational width of the scattering in the solid phase. By varying our surface preparation techniques, we have obtained solids with angular widths of from $1.4^{\circ}$ to $5^{\circ}$, far greater than the substrate mosaic of $0.16^{\circ}$. However, the ratio of the excess angular width to the radial width remains at about 4 , the value in the liquid. Thus, the low-temperature phase can be thought of as frozen hexatic phase or hexatic glass. ${ }^{5}$ A hexatic glass phase has been predicted to result from translationally invariant

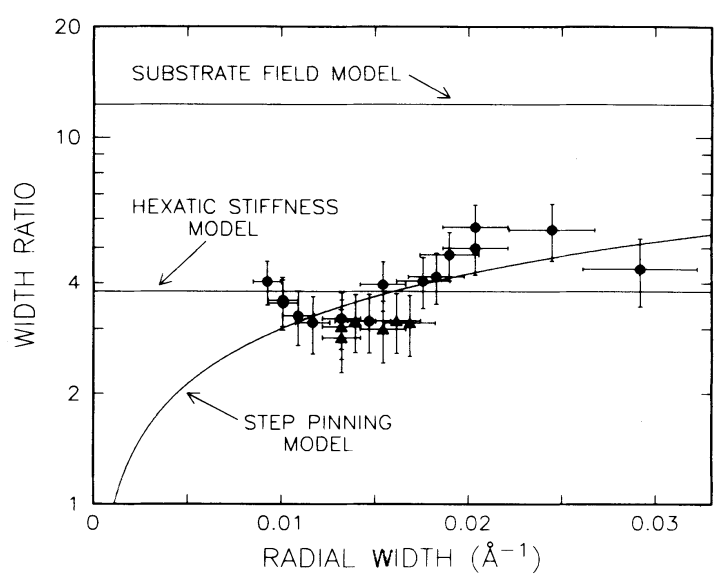

FIG. 3. Ratio of the excess angular width to the radial width, $\left(\kappa_{\theta}-\kappa\right) / \kappa$, vs the radial width $\kappa$. Open circles: $T$ increasing; closed circles: $T$ decreasing. The error bars include estimates of both statistical and systematic errors. The solid lines are theoretical fits as described in the text. 
defect sites on the surface. ${ }^{17}$ In the present situation, a more plausible source of disorder is dislocations which become pinned to grain boundaries or steps. An adlayer pinned by defects which are incommensurate with respect to the adlayer reciprocal lattice is isomorphic to a spin system in a random magnetic field. ${ }^{18}$ Spin systems in random magnetic fields usually have low-temperature Lorentzian-squared or Lorentzian-three-halves-power line shapes. ${ }^{19}$ Thus, pinning of dislocations to steps provides a possible explanation for both the low-temperature linewidths and line shapes. Furthermore, this scenario explains the large angular widths which are commonly observed for incommensurate monolayers.

In conclusion, we have studied the two-dimensional melting of xenon on silver (111). Slightly above the melting temperature, the xenon fluid has large positional correlation lengths and substantial hexatic stiffness, yielding well-defined diffraction spots. The shapes of the spots are the same as those observed for xenon on graphite in spite of the fact that the substrate is substantially weaker in the present case. Thus, hexatic order appears to be intrinsic to the xenon film. For silver, surface steps appear to determine the orientation of the xenon layer, but not to generate the observed hexatic order.

We would like to thank L. Bruch, D. DiVincenzo, G. Grinstein, D. Mukamel, and J. Toner for numerous stimulating discussions. We would also like to thank A. Schrott and M. Yu for help in sample characterization and W. Haag and J. Collett for technical assistance.

\footnotetext{
(a) Permanent address: HASYLAB, DESY, D-2000 Hamburg 52, West Germany.

${ }^{1}$ J. M. Kosterlitz and D. J. Thouless, J. Phys. C 5, L124 (1972), and 6, 1181 (1973); D. R. Nelson and B. I. Halperin, Phys. Rev. B 19, 2457 (1979); A. P. Young, Phys. Rev. B 19,
}

1855 (1979).

${ }^{2}$ See, for example, J. D. Brock et al., Phys. Rev. Lett. 57, 98 (1986).

${ }^{3}$ C. A. Murray and D. H. Van Winkle, Phys. Rev. Lett. 58, $1200(1987)$.

${ }^{4}$ For a recent review, see R. J. Birgeneau and P. M. Horn, Science 232, 329 (1986); also see T. F. Rosenbaum, S. E. Nagler, P. M. Horn, and R. Clarke, Phys. Rev. Lett. 50, 1589 (1984).

${ }^{5}$ S. E. Naglar et al., Phys. Rev. B 32, 7373 (1985); E. D. Specht et al., J. Phys. (Paris), Lett. 45, 1561 (1985).

${ }^{6}$ N. J. Colella and R. M. Suter, Phys. Rev. B 34, 2052 (1986).

${ }^{7}$ S. T. Chui, Phys. Rev. B 28, 1978 (1983); H. Kleinert, Phys. Lett. 95A, 381 (1983).

${ }^{8}$ F. F. Abraham, Phys. Rev. Lett. 50, 978 (1983); S. W. Koch and F. F. Abraham, Phys. Rev. B 27, 2964 (1983).

9. P. McTaque and A. D. Novaco, Phys. Rev. B 19, 5299 (1979).

${ }^{10}$ J. Urguris, L. W. Bruch, E. Moog, and M. B. Webb, Surf. Sci. 87, 415 (1979); P. I. Cohen, J. Unguris, and M. B. Webb, Surf. Sci. 58, 429 (1976).

${ }^{11}$ L. W. Bruch and J. M. Phillips, Surf. Sci. 91, 1 (1980); J. M. Phillips, L. W. Bruch, and R. D. Murphy, J. Chem. Phys. 75, 5097 (1981).

${ }^{12}$ G. Boato, P. Cantini, and R. Tatarek, Phys. Rev. Lett. 40, 887 (1978); K. Kern et al., Phys. Rev. Lett. 57, 3187 (1986).

${ }^{13}$ P. Dimon, P. M. Horn, M. Sutton, R. J. Birgeneau, and D. E. Moncton, Phys. Rev. B 31, 437 (1985).

${ }^{14} \mathrm{~L}$. W. Bruch, private communication.

${ }^{15} L$ is determined from the in-plane radial width of the $\mathrm{Ag}(1 \overline{1} l)$ crystal truncation rod near $l=0$.

${ }^{16} \mathrm{~A}$ crude estimate for $E_{p}$ can be obtained by assuming that a $30^{\circ}$ grain boundary approximately costs an energy of $20 \%$ of the xenon-xenon Lennard-Jones attraction; D. DiVincenzo, private communication.

${ }^{17}$ D. R. Nelson, Phys. Rev. B 27, 2909 (1983).

${ }^{18}$ Y. Imry and S.-k. Ma, Phys. Rev. Lett. 35, 1399 (1975).

${ }^{19}$ R. J. Birgeneau, H. Yoshizawa, R. A. Cowley, G. Shirane, and H. Ikeda, Phys. Rev. B 28, 1433 (1983). 